\title{
Dislipidemia e fatores associados em adultos residentes em Ribeirão Preto, SP. Resultados do Projeto EPIDCV
}

\author{
Dyslipidemia and correlates in adults living in \\ Ribeirõo Preto, SP. Results of the EPIDCV Project
}

Suzana Alves de Moraes', Michele Vantini Checchio², Isabel Cristina Martins de Freitas'

\begin{abstract}
RESUMO
Objetivo: Identificar a prevalência de dislipidemia e fatores associados em adultos residentes em Ribeirão Preto, SP, em 2007. Materiais e métodos: Estudo epidemiológico transversal de base populacional, com amostragem desenvolvida em três estágios. A variabilidade introduzida na terceira fração foi corrigida pela atribuição de pesos, originando amostra ponderada de 2.471 participantes. Prevalências da dislipidemia, definida como alteração de pelo menos uma, entre quatro frações lipídicas, foram estimadas em ambos os sexos, segundo variáveis sociodemográficas, comportamentais e relacionadas à saúde. Para identificar fatores associados, utilizou-se a regressão de Poisson, obtendo-se razões de prevalências em modelos uni- e multivariados. Todas as estimativas foram calculadas levando-se em consideração o efeito de desenho amostral. Resultados: Elevada prevalência de dislipidemia foi observada no município (61,9\%; $\mathrm{IC}_{95 \%}$ : 58,5\%-65,3\%). Renda e consumo de fibras, no sexo masculino, e média diária de tempo sentado e consumo de ácidos graxos saturados, no feminino, se mantiveram nos modelos finais, além da idade e dos indicadores de obesidade central que permaneceram consistentemente associados ao desfecho, em ambos os sexos. Conclusões: Políticas públicas de intervenção direcionadas à adoção de hábitos saudáveis de consumo alimentar, bem como ao controle da obesidade central e do sedentarismo, poderão contribuir para o declínio da prevalência de dislipidemia e, por conseguinte, da morbimortalidade por doenças cardiovasculares no município. Arq Bras Endocrinol Metab. 2013;57(9):691-701
\end{abstract}

\section{Descritores}

Dislipidemia; fatores associados; estudos transversais; epidemiologia; saúde pública

\begin{abstract}
Objective: To identify the prevalence and correlates of dyslipidemia in adults living in the city of Ribeirão Preto, SP, Brazil, in 2007. Materials and methods: A cross-sectional population-based epidemiological study was carried out using a three-stage cluster sampling. The variability introduced in the third sampling fraction was corrected, resulting in a weighted sample of 2,471 participants. Dyslipidemia prevalence, defined as abnormal values in at least one of four lipid fractions was estimated in both genders, according sociodemographic, behavioral, and health-related variables. To identify correlates, prevalence ratios were estimated using Poisson regression, in crude and adjusted models. All the estimates were calculated taking into account the sampling design effect. Results: High prevalence of dyslipidemia was found $\left(61.9 \% ; \mathrm{Cl}_{95 \%}: 58.5 \%-65.3 \%\right)$. Income and fiber consumption, in males, and sitting time and saturated fatty acids, in females, were kept in the final models, as well as age and central obesity, which were consistently associated with the outcome, in both genders. Conclusions: Public intervention policies toward the adoption of healthy food consumption, as well as control central obesity and sedentary behavior might contribute to decrease the prevalence of dyslipidemia and cardiovascular morbidity and mortality in Ribeirão Preto. Arq Bras Endocrinol Metab. 2013;57(9):691-701
\end{abstract}

\section{Keywords}

Dyslipidemia; correlates; cross-sectional studies; epidemiology; public health
1 Departamento de Enfermagem Materno-Infantil e Saúde Pública, Escola de Enfermagem de Ribeirão Preto, Universidade de São Paulo, Núcleo de Epidemiologia (EERPUSP/NEPI), Ribeirão Preto, SP, Brasil 2 Programa de Pós-Graduação em Enfermagem e Saúde Pública, EERP-USP/NEPI, Ribeirão Preto, SP, Brasil

Correspondência para: Suzana Alves de Moraes Av. Bandeirantes, 3900 14040-902 - Campus USP Ribeirão Preto, SP, Brasil samoraes@usp.br

Recebido em 28/Mar/2013 Aceito em 19/Ago/2013 


\section{INTRODUÇÃO}

$\mathrm{O}$ crescente aumento das doenças crônico-degenerativas, em detrimento das infecciosas, ao lado da inversão do padrão desnutrição/excesso de peso, configura um cenário presente na maioria dos países desenvolvidos ou em desenvolvimento (1).

Nesse contexto, as doenças cardiovasculares (DCV) têm sido apontadas como um importante desfecho crônico, representando $30 \%$ do total de mortes no mundo (2). Estudos epidemiológicos têm relatado declínio da mortalidade por esse grupo de causas, característica típica de países que passaram ou estão passando pelo quarto estágio da transição epidemiológica, embora o envelhecimento populacional progressivo aponte para aumento da incidência e da prevalência das $\operatorname{DCV}(3)$.

Entre os fatores de risco associados às $\mathrm{DCV}$, encontram-se as dislipidemias. Caracterizadas por concentrações anormais de lipídios ou lipoproteínas no sangue, favorecem o desenvolvimento da aterosclerose (4).

Entre os fatores associados às dislipidemias, determinantes sociodemográficos como a escolaridade e a renda, bem como fatores comportamentais, dietéticos, estado nutricional e indicadores de inflamação, vêm ganhando destaque (5-8).

Considerando-se a relevância dos fatores supramencionados sobre o perfil lipídico, o presente estudo teve por objetivo investigar a prevalência de dislipidemia e fatores associados em adultos residentes em Ribeirão Preto, SP.

\section{MATERIAIS E MÉTODOS}

\section{Delineamento do estudo e processo de amostragem}

O estudo apresenta delineamento transversal, sendo parte integrante do Projeto EPIDCV - "Prevalência de doenças cardiovasculares e identificação de fatores associados em adultos residentes em Ribeirão Preto, SP, 2006", estudo epidemiológico de base populacional conduzido no município, no período 2007/2008.

$\mathrm{O}$ processo de amostragem foi desenvolvido em três estágios, e a precisão das estimativas, calculadas em amostra de 1.205 indivíduos, correspondeu a erros de amostragem fixados em torno de $2 \%$ (para prevalências abaixo de $15 \%$ ou acima de $75 \%$ ) e em torno de $3 \%$ (para prevalências entre $20 \%$ e $80 \%$ ). No primeiro estágio, 81 setores censitários foram sorteados, e, na sequência, 1.672 domicílios e 1.395 participantes, correspondendo estes últimos, respectivamente, ao segundo e terceiro estágios de amostragem. A taxa de resposta foi $81,2 \%$, o que correspondeu a 1.133 adultos participantes. A variabilidade introduzida, principalmente na terceira fração de amostragem, foi corrigida por meio do cálculo de pesos amostrais que levaram em consideração o "número de unidades elegíveis de cada domicílio" e as taxas de "não resposta", em cada setor censitário. Ao todo, 1.133 indivíduos de ambos os sexos, com idade $\geq 30$ anos e residentes em Ribeirão Preto, foram entrevistados. Gestantes e puérperas até o sexto mês foram excluídas, visto que suas medidas antropométricas não seriam comparáveis às da população. A taxa de resposta foi equivalente a $81,2 \%$. As perdas $(18,8 \%)$ foram decorrentes de mudança de endereço $(4,8 \%)$; óbitos $(0,5 \%)$ e recusas $(13,5 \%)$, estas últimas, consideradas como tal, após cinco tentativas de contato para a entrevista, em dias e períodos alternados. O estudo foi desenvolvido em amostra ponderada (nw) de 2.471 participantes (9). As estimativas populacionais estiveram baseadas no Censo 2000 (10).

\section{Variáveis do estudo}

\section{Variável dependente}

Dislipidemia: amostras de sangue venoso foram obtidas após jejum de 12 horas para dosagens séricas $(\mathrm{mg} / \mathrm{dL})$ dos lipídios: CT, TG, LDL-c e HDL-c que foram processadas em Laboratório de Referência com Certificado de Proficiência em Ensaios Laboratoriais. A variável dislipidemia foi gerada de forma dicotômica. Todos os participantes que apresentaram anormalidades em pelo menos um dos lipídios, conforme recomendações do National Cholesterol Education Program NCEP-ATPIII (11): colesterol total $(\mathrm{CT}) \geq 200 \mathrm{mg} / \mathrm{dL}$; lipoproteínas de alta densidade (HDLc) $<40 \mathrm{mg} / \mathrm{dL}$; lipoproteínas de baixa densidade (LDLc) $\geq 130 \mathrm{mg} / \mathrm{dL}$ ou triglicérides (TG) $>150 \mathrm{mg} / \mathrm{dL}$, foram classificados na categoria ("sim") e os que apresentaram valores normais para todos esses componentes, classificados como referência ("nã 0 ").

\section{Variáveis independentes}

Variáveis sociodemográficas: incluíram-se participantes de ambos os sexos, com 30 anos ou mais. A escolaridade foi classificada segundo o número de anos completos de escolaridade formal. A renda individual (em reais) teve como referência o mês que antecedeu as entrevistas. Os que não referiram renda, no período, foram classificados como "sem renda". O estado marital foi definido, segundo a presença de companheiro(a), independentemente de união formal. Os participantes 
também foram classificados em duas categorias (não/ sim), segundo inserção no mercado de trabalho formal, no mês que antecedeu a entrevista.

Variáveis comportamentais: o padrão de atividade física (PAF) e a média diária de tempo sentado foram obtidos a partir do International Physical Activity Questionnaire (IPAQ), versão curta (12). O bábito de fumar foi classificado em três categorias (não fumantes, ex-fumantes e fumantes), considerando-se, entre os fumantes, a duração do hábito. O padrão alimentar foi definido por meio da aplicação de um Questionário de Frequência de Consumo Alimentar (QFCA), contendo 128 itens. O cálculo das respectivas quantidades de micro- e macronutrientes foi efetuado no aplicativo NUTWIN, mediante a utilização de tabelas específicas, considerando-se a composição centesimal de cada alimento, o tipo de preparação e as medidas caseiras utilizadas. A quantidade de alimento/dia foi resultante do cálculo (escores de consumo x número de porções), utilizandose para o cálculo dos escores o modelo proposto por Fornés e cols. (13). No presente estudo, consideraramse o consumo diário de ácidos graxos saturados (gramas/dia), o consumo de fibras (gramas/dia) e a energia total da dieta $(\mathrm{kcal} / \mathrm{dia})$, classificados segundo os respectivos tercis das distribuições. $\mathrm{O}$ consumo diário de ácidos graxos saturados e o consumo de fibras foram ajustados para a energia total da dieta pelo método do resíduo proposto por Willett (14). O QFCA foi aplicado por entrevistadores treinados por uma nutricionista com larga experiência em Epidemiologia Nutricional.

Variáveis relacionadas à saúde: indicadores antropométricos: a obesidade global foi classificada em três categorias: "eutróficos", "com sobrepeso" e "obesos", segundo pontos de corte para o índice de massa corporal (IMC), definidos pela Organização Mundial da Saúde (OMS) (15). O peso foi aferido em balanças eletrônicas da marca TANITA, modelo BF 680, com precisão de 100 gramas. A altura foi aferida em estadiômetros de parede (SECA, Hamburgo, Alemanha). A obesidade central foi definida utilizando-se três indicadores: 1) Circunferência da Cintura (CC) (cm), utilizando-se como referência a menor curvatura entre o rebordo costal e a crista ilíaca. A variável foi classificada em duas categorias (normal e alterada), segundo pontos de corte específicos para o sexo $(16) ; 2$ ) Razão Cintura-Quadril (RCQ), adotando-se como referência para a circunferência do quadril a maior protuberância dos glúteos, classificada segundo tercis e 3) Índice de conicidade (17), obtido por meio da fórmula:

$$
\text { Índice de conicidade }=\frac{\text { Cincunferência da cintura }}{0,109 \sqrt{\frac{\text { Peso em kg }}{\text { Altura em metros }}}}
$$

classificado segundo tercis. As circunferências utilizadas como medidas de obesidade central foram aferidas por meio de fitas inextensíveis (SECA, Hamburgo, Alemanha).

Acesso a serviços de saúde: além das medidas antropométricas, considerou-se também o acesso a serviços ambulatoriais de saúde, nos seis meses que antecederam as entrevistas, sendo a variável classificada de forma dicotômica (não/sim).

Dosagens bioquimicas: dosagens de proteína C reativa-ultrassensível (PCR-U), em mg/dL, foram obtidas após 12 horas de jejum, sendo a variável classificada em duas categorias: normal e alterada (PCR-U $>0,5 \mathrm{mg} /$ $\mathrm{dL}$ ), conforme o ponto de corte recomendado pelo Laboratório de Referência. Os participantes que desconheciam ser portadores de diabetes foram submetidos a dosagens glicêmicas, após jejum de 12 horas, e também duas horas após a sobrecarga com solução contendo 75 gramas de glicose pura, utilizando-se os pontos de corte recomendados pela OMS para a classificação de diabetes (18). No presente estudo, foram classificados como diabéticos os que preencheram esses critérios, além daqueles com diagnóstico prévio dessa condição, no momento da entrevista.

Processamento dos dados: a coleta de dados foi realizada mediante a aplicação de entrevistas estruturadas, aplicadas no domicílio dos elegíveis por equipe de entrevistadores previamente treinada. Antes da digitação definitiva, processada com dupla entrada de dados, o controle de qualidade das informações foi avaliado por meio da replicação de $12,5 \%$ do total de entrevistas. Como medida de reprodutibilidade para as variáveis incluídas na replicação, utilizou-se a estatística Kappa que alcançou valores superiores a 0,80 .

Análise estatística: 1) Fase descritiva: as prevalências de dislipidemias foram estimadas por pontos e por intervalos com 95\% de confiança, segundo variáveis sociodemográficas, comportamentais e relacionadas à saúde. Nesta fase, associações globais foram definidas a partir dos respectivos valores da estatística "F" adotando-se nível de significância $\alpha=0,05$. 2) Fase analítica: na fase analítica, utilizou-se a regressão de Poisson (19) para obtenção das razões de prevalências brutas e ajustadas que foram estimadas por pontos e por intervalos com 95\% de confiança. Os modelos finais foram compostos pelo elenco de variáveis que, após ajustamento simultâ- 
neo, mantiveram valores " $p$ " $<0,05$. Todas as análises foram desenvolvidas no software Stata versão 10.1 para Windows. O cálculo de todas as estimativas levou em consideração o efeito de desenho amostral (deff), utilizando-se comandos específicos do Stata.

Considerações éticas: o Projeto EPIDCV foi aprovado pelo Comitê de Ética em Pesquisa da Escola de Enfermagem de Ribeirão Preto da Universidade de São Paulo e protocolado sob o $\mathrm{n}^{\mathrm{o}}$. $0725 / 2006$. Todos os participantes assinaram o Termo de Consentimento Livre e Esclarecido, conforme recomendações da Resolução ${ }^{\circ}$. 196/96 do Conselho Nacional de Saúde.

\section{RESULTADOS}

Na tabela 1 , apresenta-se a caracterização da amostra, segundo variáveis sociodemográficas e perfil lipídico. A maioria foi composta por participantes com alta escola-

Tabela 1. Características da população do estudo, segundo fatores sociodemográficos e dislipidemia. Ribeirão Preto, São Paulo, Brasil, 2007. Projeto EPIDCV

\begin{tabular}{|c|c|c|c|c|}
\hline Variáveis & $\mathbf{N}_{\mathbf{w}}$ & $\%$ & Medianas & $\mathbf{I C}_{95 \%}$ \\
\hline \multicolumn{5}{|l|}{ Sexo } \\
\hline Masculino & 995,7 & 40,3 & & {$[37,3-43,4]$} \\
\hline Feminino & 1.475 & 59,7 & & {$[56,6-62,7]$} \\
\hline Faixas etárias & & & 47,1 & {$[46,3-48,0]$} \\
\hline 30-39 anos & 661,3 & 26,8 & & {$[23,4-30,4]$} \\
\hline 40-49 anos & 765,7 & 31,0 & & {$[28,3-33,8]$} \\
\hline $50-59$ anos & 507,3 & 20,5 & & {$[18,3-23,0]$} \\
\hline 60 anos e mais & 536,6 & 21,7 & & {$[18,8-24,9]$} \\
\hline \multicolumn{5}{|l|}{ Escolaridade } \\
\hline 0-3 anos & 351,6 & 14,2 & & {$[11,7-17,2]$} \\
\hline 4-7 anos & 749,1 & 30,3 & & {$[26,7-34,2]$} \\
\hline 8 anos e mais & 1.370 & 55,5 & & {$[50,3-60,5]$} \\
\hline Renda individual (em R\$) & & & $1.000,00$ & {$[950,00-1.000,01]$} \\
\hline Sem renda & 848,9 & 34,3 & & {$[31,6-37,2]$} \\
\hline $1^{\circ}$ terço $(50,00-700,00)$ & 549,7 & 22,2 & & {$[19,3-25,5]$} \\
\hline $2^{\circ}$ terço $(700,00-1.400,00)$ & 506 & 20,6 & & {$[18,0-23,2]$} \\
\hline $3^{\circ}$ terço $(>1.400,00)$ & 566,4 & 22,9 & & {$[19,2-27,1]$} \\
\hline \multicolumn{5}{|l|}{ Estado marital } \\
\hline Sem companheiro(a) & 824,8 & 33,4 & & {$[30,1-36,9]$} \\
\hline Com companheiro(a) & 1646 & 66,6 & & {$[63,1-69,9]$} \\
\hline \multicolumn{5}{|l|}{ Condição de trabalho } \\
\hline Não & 847,8 & 34,3 & & {$[31,5-37,2]$} \\
\hline $\operatorname{Sim}$ & 1.623 & 65,7 & & {$[62,8-68,4]$} \\
\hline Colesterol total (em mg/dL) & & & 197,6 & {$[196,5-198,9]$} \\
\hline alterado ( $\geq 200$ mg/dL) & 1.080 & 43,7 & & {$[40,5-47,0]$} \\
\hline LDLc (em mg/dL) & & & 124,2 & {$[123,5-125,3]$} \\
\hline alterado ( $\geq 130$ mg/dL) & 822,4 & 33,3 & & {$[30,5-36,1]$} \\
\hline HDLc (em mg/dL) & & & 46,0 & {$[45,0-46,9]$} \\
\hline alterado (< 40 mg/dL) & 503,8 & 20,4 & & {$[17,5-23,2]$} \\
\hline Triglicerídeos (em mg/dL) & & & 123,0 & {$[120,2-128,0]$} \\
\hline alterado ( $\geq 150$ mg/dL) & 617 & 25,0 & & {$[22,3-27,6]$} \\
\hline \multicolumn{5}{|l|}{ Dislipidemia* } \\
\hline Não & 940,2 & 38,0 & & {$[34,7-41,5]$} \\
\hline Sim & 1530,8 & 61,9 & & {$[58,5-65,3]$} \\
\hline
\end{tabular}

* Alteração em pelo menos 1 entre 4 frações lipídicas. 
ridade, que vivia com companheiro(a) e estava inserida no mercado de trabalho. As prevalências de alterações das frações lipídicas oscilaram entre $20,4 \%$ e $43,7 \%$, e as respectivas medianas estiveram próximas dos pontos de corte limítrofes. A prevalência de dislipidemia foi $61,9 \%$ (efeito de desenho- $\operatorname{deff}=1,43341$ ).

Na tabela 2, prevalências de dislipidemia, estratificadas por sexo, são apresentadas, segundo categorias dos fatores sociodemográficos. Em ambos os sexos, a prevalência de dislipidemia aumentou com a idade, sugerindo gradiente linear, enquanto para as variáveis "escolaridade" e "renda" as respectivas prevalências declinaram com o aumento do número de anos de escolaridade e os terços de renda. Em ambos os sexos, associação global foi identificada para as variáveis "faixa etária" e "estado marital".

Nas tabelas 3 e 4, as prevalências de dislipidemia, estratificadas por sexo, são apresentadas segundo categorias de comportamentais e fatores relacionados à saúde. Em ambos os sexos, a prevalência de dislipidemia foi maior no último terço da "média diária de tempo sentado" e da "duração do hábito de fumar"; entre aqueles que consumiram mais de $24,7 \mathrm{~g} /$ dia de fibras; com dietas abaixo de $2.800 \mathrm{kcal} /$ dia e que consumiram menos de $25 \mathrm{~g} /$ dia de ácidos graxos saturados. No sexo masculino, associação global com o desfecho foi identificada para a variável "consumo de fibras", e, no feminino, para as variáveis "média diária de tempo sentado", "duração do hábito de fumar", "energia total da dieta" e "ácidos graxos saturados" (Tabela 3). Em relação aos fatores relacionados à saúde (Tabela 4), destacaram-se, em ambos os sexos, prevalências do desfecho de maior magnitude, entre os que apresentaram níveis alterados de "PCR-U"; nos "diabéticos"; naqueles com excesso de peso (IMC $\geq$ $25 \mathrm{~kg} / \mathrm{m}^{2}$ ) e a partir do segundo terço dos indicadores de obesidade central. A variável "PCR-U" apresentou associação global com o desfecho, no sexo masculino, e a variável "diabetes", no feminino.

Tabela 2. Prevalências de dislipidemia e respectivos intervalos de confiança $\left(I_{9_{55}}\right)$, segundo sexo e fatores sociodemográficos. Ribeirão Preto, São Paulo, Brasil, 2007. Projeto EPIDCV

\begin{tabular}{|c|c|c|c|c|}
\hline & \multicolumn{2}{|c|}{ Masculino } & \multicolumn{2}{|c|}{ Feminino } \\
\hline & \multicolumn{2}{|c|}{ Dislipidemia } & \multicolumn{2}{|c|}{ Dislipidemia } \\
\hline & $\begin{array}{c}\text { Não } \\
\left.\text { \% [IC }{ }_{95 \%}\right]\end{array}$ & $\operatorname{Sim}_{\%\left[\mathrm{IC}_{95 \%}\right]}$ & $\begin{array}{c}\text { Não } \\
\text { \% }\left[\text { [C }_{95 \%}\right]\end{array}$ & $\operatorname{Sim}_{\%\left[\mathrm{IC}_{95 \%}\right]}$ \\
\hline \multicolumn{5}{|l|}{ Faixas etárias ${ }^{\dagger \ddagger}$} \\
\hline 30-39 anos & $57,9[48,8-66,4]$ & $42,1[33,6-51,2]$ & $66,0[57,2-73,8]$ & $34,0[26,2-42,8]$ \\
\hline 40-49 anos & $43,1[34,1-52,6]$ & $56,9[47,4-65,9]$ & $40,5[34,2-47,1]$ & $59,5[52,9-65,8]$ \\
\hline $50-59$ anos & $33,2[23,4-44,8]$ & $66,8[55,2-76,6]$ & $16,9[11,6-24,0]$ & $83,1[76,0-88,4]$ \\
\hline 60 anos e mais & $15,7[9,6-24,6]$ & $84,3[75,4-90,4]$ & $19,6[13,5-27,5]$ & $80,4[72,4-86,5]$ \\
\hline \multicolumn{5}{|l|}{ Escolaridade $^{\ddagger}$} \\
\hline $0-3$ anos & $39,8[28,7-52,1]$ & $60,2[47,9-71,3]$ & $23,3[15,6-33,3]$ & $76,7[66,7-84,4]$ \\
\hline 4-7 anos & $41,3[32,3-50,9]$ & $58,7[49,1-67,7]$ & $29,8[23,6-36,9]$ & $70,2[63,0-76,4]$ \\
\hline 8 anos e mais & $41,0[35,0-47,3]$ & $59,0[52,6-65,0]$ & $43,3[37,9-48,9]$ & $56,7[51,1-62,1]$ \\
\hline \multicolumn{5}{|l|}{ Renda individual $(e m \mathrm{R} \$)^{\ddagger}$} \\
\hline Sem renda & $35,3[26,7-45,0]$ & $64,7[55,0-73,3]$ & $30,0[24,1-36,7]$ & $70,0[63,3-75,9]$ \\
\hline $1^{\circ}$ terço $(\leq 700,00)$ & $44,6[32,7-57,1]$ & $55,4[42,9-67,3]$ & $38,2[31,3-45,7]$ & $61,8[54,3-68,7]$ \\
\hline $2^{\circ}$ terço $(>700,00 \leq 1.400,00)$ & $37,0[28,6-46,2]$ & $63,0[53,8-71,4]$ & $43,9[35,2-52,9]$ & $56,1[47,0-64,8]$ \\
\hline $3^{\circ}$ terço $(>1.400,00)$ & $45,3[37,5-53,3]$ & $54,7[46,7-62,5]$ & $44,2[33,4-55,6]$ & $55,8[44,4-66,6]$ \\
\hline \multicolumn{5}{|l|}{ Estado maritall ${ }^{\dagger \ddagger}$} \\
\hline Sem companheiro(a) & $48,6[39,8-57,3]$ & $51,4[42,6-60,1]$ & $28,7[23,4-34,7]$ & $71,3[65,3-76,6]$ \\
\hline Com companheiro(a) & $38,3[32,9-44,1]$ & $61,7[55,9-67,1]$ & $40,7[35,5-46,1]$ & $59,3[53,9-64,5]$ \\
\hline \multicolumn{5}{|l|}{ Condição de trabalho $0^{\ddagger}$} \\
\hline Não & $35,6[27,1-45,2]$ & $64,4[54,8-72,9]$ & $30,0[24,1-36,6]$ & $70,0[63,4-75,9]$ \\
\hline Sim & $42,1[36,8-47,6]$ & $57,9[52,4-63,2]$ & $41,2[35,9-46,8]$ & $58,8[53,2-64,1]$ \\
\hline
\end{tabular}

${ }^{\dagger}$ valor $\mathrm{p}<0,05$ para a estatística $\mathrm{F}$ - sexo masculino; ₹ valor $\mathrm{p}<0,05$ para a estatística $\mathrm{F}$ - sexo feminino. 
Tabela 3. Prevalência de dislipidemia e respectivos intervalos de confiança $\left(\mathrm{IC}_{95 \%}\right)$, segundo sexo e fatores comportamentais. Ribeirão Preto, São Paulo, Brasil, 2007. Projeto EPIDCV

\begin{tabular}{|c|c|c|c|c|}
\hline & \multicolumn{2}{|c|}{ Masculino } & \multicolumn{2}{|c|}{ Feminino } \\
\hline & \multicolumn{2}{|c|}{ Dislipidemia } & \multicolumn{2}{|c|}{ Dislipidemia } \\
\hline & $\begin{array}{c}\text { Não } \\
\left.\text { \% [IC }{ }_{95 \%}\right]\end{array}$ & $\operatorname{Sim}_{\%\left[I C_{95 \%}\right]}$ & $\begin{array}{c}\text { Não } \\
\text { \% }\left[\mathrm{IC}_{95 \%}\right]\end{array}$ & $\operatorname{Sim}_{\%\left[\mathrm{IC}_{95 \%}\right]}$ \\
\hline \multicolumn{5}{|c|}{ Média diária de tempo sentado (minutos/dia) ${ }^{\ddagger}$} \\
\hline $1^{\circ}$ terço $(\leq 180,0)$ & $43,1[35,7-50,8]$ & $56,9[49,2-64,3]$ & $38,4[31,9-45,3]$ & $61,6[54,7-68,1]$ \\
\hline $2^{\circ}$ terço $(>180,0 \leq 308,6)$ & $39,3[31,8-47,3]$ & $60,7[52,7-68,2]$ & $42,8[35,8-50,0]$ & $57,2[50,0-64,2]$ \\
\hline $3^{\circ}$ terço $(>308,6)$ & $40,6[33,8-47,9]$ & $59,3[52,1-66,2]$ & $27,8[22,4-33,9]$ & $72,2[66,1-77,6]$ \\
\hline \multicolumn{5}{|l|}{ Padrão de atividade física } \\
\hline Muito ativos & $17,8[7,0-38,4]$ & $82,1[61,6-93,0]$ & $46,0[27,1-66,2]$ & $54,0[33,8-72,9]$ \\
\hline Moderadamente ativos & $45,0[36,3-54,0]$ & $55,0[46,0-63,7]$ & $35,4[29,0-42,4]$ & $64,6[57,6-71,0]$ \\
\hline Pouco ativos & $40,9[34,3-47,8]$ & $59,1[52,2-65,7]$ & $35,8[31,4-40,6]$ & $64,1[59,4-68,6]$ \\
\hline \multicolumn{5}{|c|}{ Duração do hábito de fumar (em anos) ${ }^{\ddagger}$} \\
\hline Não fumantes & $38,9[31,2-47,2]$ & $61,1[52,8-68,7]$ & $36,7[31,5-42,2]$ & $63,3[57,8-68,5]$ \\
\hline $1^{\circ}$ terço $(\leq 13)$ & $50,2[40,9-59,5]$ & $49,8[40,5-59,1]$ & $42,0[32,3-52,4]$ & $58,0[47,6-67,7]$ \\
\hline $2^{\circ}$ terço $(>13 \leq 25)$ & $44,8[33,6-56,5]$ & $55,2[43,5-66,4]$ & $44,6[33,5-56,3]$ & $55,4[43,7-66,5]$ \\
\hline $3^{\circ}$ terço $(>25)$ & $31,6[21,1-44,3]$ & $68,4[55,7-78,9]$ & $16,8[9,8-27,2]$ & $83,2[72,8-90,2]$ \\
\hline \multicolumn{5}{|l|}{ Energia total $(\mathrm{em} \mathrm{kcal} / \mathrm{dia})^{\ddagger}$} \\
\hline $1^{\circ}$ terço $(\leq 1.924,8)$ & $40,5[27,3-55,3]$ & $59,5[44,7-72,7]$ & $31,4[25,8-37,5]$ & $68,6[62,4-74,2]$ \\
\hline $2^{\circ}$ terço $(>1.924,8 \leq 2.772,4)$ & $36,5[28,4-45,5]$ & $63,5[54,4-71,6]$ & $36,0[29,2-43,5]$ & $64,0[56,5-70,8]$ \\
\hline $3^{\circ}$ terço $(>2.772,4)$ & $43,9[37,3-50,7]$ & $56,1[49,3-62,7]$ & $47,1[39,0-55,3]$ & $52,9[44,7-61,0]$ \\
\hline \multicolumn{5}{|c|}{ Ácidos graxos saturados (em gramas/dia) ${ }^{\ddagger}$} \\
\hline $1^{\circ}$ terço $(\leq 19,2)$ & $35,2[28,5-42,5]$ & $64,8[57,5-71,5]$ & $30,4[23,6-38,3]$ & $69,5[61,7-76,4]$ \\
\hline $2^{\circ}$ terço $(>19,2 \leq 24,8)$ & $41,3[31,7-51,5]$ & $58,7[48,5-68,3]$ & $32,4[26,8-38,6]$ & $67,6[61,4-73,2]$ \\
\hline $3^{\circ}$ terço $(>24,8)$ & $48,4[38,6-58,4]$ & $51,6[41,6-61,4]$ & $43,8[37,9-50,0]$ & $56,2[50,0-62,1]$ \\
\hline \multicolumn{5}{|l|}{ Consumo de fibras (em gramas/dia) ${ }^{\dagger}$} \\
\hline $1^{\circ}$ terço $(\leq 24,7)$ & $48,5[38,4-58,7]$ & $51,5[41,3-61,6]$ & $31,8[26,5-37,6]$ & $68,2[62,4-73,5]$ \\
\hline $2^{\circ}$ terço $(>24,7 \leq 42,2)$ & $32,7[26,0-40,2]$ & $67,3[59,8-74,0]$ & $39,4[32,7-46,6]$ & $60,6[53,4-67,3]$ \\
\hline $3^{\circ}$ terço $(>42,2)$ & $42,8[35,9-50,0]$ & $57,2[49,9-64,1]$ & $39,0[30,5-48,2]$ & $61,0[51,8-69,5]$ \\
\hline
\end{tabular}

† valor $p<0,05$ para a estatística $F-$ sexo masculino; ${ }^{\ddagger}$ valor $p<0,05$ para a estatística $F-$ sexo feminino.

Na figura 1, apresenta-se o perfil lipídico, segundo estratos da variável "diabetes", sendo semelhantes os “box-plots” das dosagens de colesterol total e frações nesses estratos.

$\mathrm{Na}$ tabela 5, são apresentados os modelos finais, estratificados por sexo, com razões de prevalências brutas, ajustadas, e respectivos intervalos de confiança. No sexo masculino, permaneceram as variáveis "faixas etárias", "renda", "consumo de fibras" e "índice de conicidade". No sexo feminino, permaneceram as variáveis "faixas etárias", "consumo de ácidos graxos saturados", "média diária de tempo sentado" e "RCQ". Em ambos os sexos, para fins de ajustamento, foram retidas as variáveis "acesso a serviços de saúde" e "diabetes".

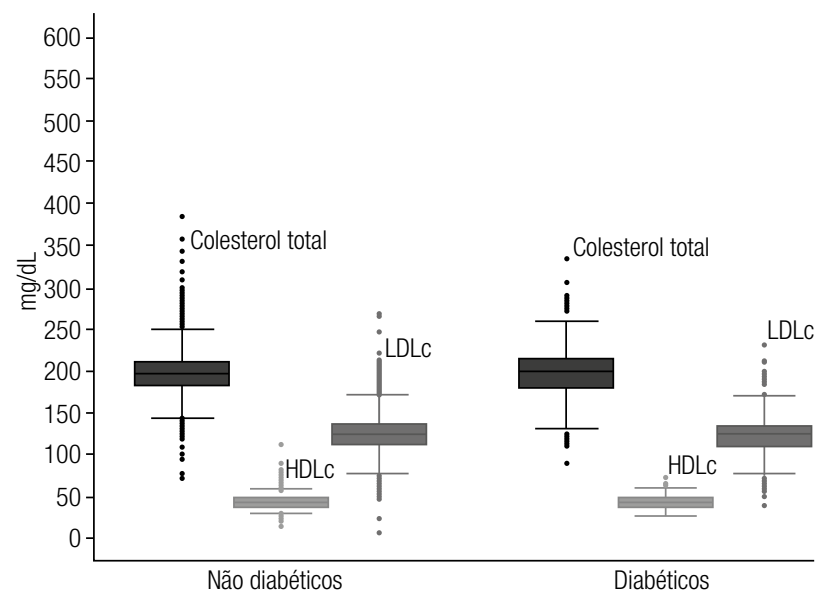

Figura 1. Perfil lipídico da população do estudo, em diabéticos e não diabéticos. Ribeirão Preto, São Paulo, Brasil, 2007. Projeto EPIDCV 
Tabela 4. Prevalência de dislipidemia e respectivos intervalos de confiança $\left(\mathrm{IC}_{95 \%}\right)$, segundo sexo e fatores relacionados à saúde. Ribeirão Preto, São Paulo, Brasil, 2007. Projeto EPIDCV

\begin{tabular}{|c|c|c|c|c|}
\hline & \multicolumn{2}{|c|}{ Masculino } & \multicolumn{2}{|c|}{ Feminino } \\
\hline & \multicolumn{2}{|c|}{ Dislipidemia } & \multicolumn{2}{|c|}{ Dislipidemia } \\
\hline & $\begin{array}{c}\text { Não } \\
\text { \% }\left[\mathrm{IC}_{95 \%}\right]\end{array}$ & $\underset{\text { \% }}{\operatorname{Sim}}$ & 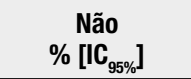 & $\underset{\text { \% }}{\operatorname{Sim}}$ \\
\hline \multicolumn{5}{|l|}{ Estado nutricional| ${ }^{\ddagger}$} \\
\hline Eutrófico (IMC > 18 e < 25) & $52,0[43,0-60,9]$ & $48,0[39,1-57,0]$ & $44,6[37,9-51,4]$ & $55,4[48,6-62,1]$ \\
\hline Com sobrepeso (IMC $\geq 25$ e $<30)$ & $38,7[31,3-46,6]$ & $61,3[53,4-68,7]$ & $31,8[25,3-39,1]$ & $68,2[60,9-74,7]$ \\
\hline Obeso (IMC $\geq 30$ ) & $27,9[19,8-37,7]$ & $72,1[62,3-80,2]$ & $30,4[23,7-38,1]$ & $69,6[61,9-76,3]$ \\
\hline \multicolumn{5}{|l|}{ Circunferência da cintura ${ }^{\star \star \ddagger}$} \\
\hline Normal & $52,1[45,0-59,1]$ & $47,9[40,9-55,0]$ & $50,1[42,6-57,7]$ & $49,9[42,3-57,4]$ \\
\hline Alterada & $30,0[24,2-36,5]$ & $70,0[63,5-75,8]$ & $30,3[25,6-35,5]$ & $69,7[64,5-74,4]$ \\
\hline \multicolumn{5}{|l|}{ Razão cintura-quadril|t } \\
\hline $1^{\circ}$ terço $(\leq 0,8)$ & $53,7[45,3-61,9]$ & $46,3[38,1-54,7]$ & $53,6[46,9-60,2]$ & $46,4[39,84-53,08]$ \\
\hline $2^{\circ}$ terço $(>0,8 \leq 0,9)$ & $39,0[32,4-46,1]$ & $61,0[53,9-67,6]$ & $30,0[24,3-36,3]$ & $70,0[63,7-75,7]$ \\
\hline $3^{\circ}$ terço $(>0,9)$ & $30,5[23,1-39,0]$ & $69,5[61,0-76,9]$ & $24,2[17,9-31,8]$ & $75,8[68,2-82,1]$ \\
\hline \multicolumn{5}{|l|}{ Índice de conicidade ${ }^{\dagger \ddagger}$} \\
\hline $1^{\circ}$ terço $(\leq 1,2)$ & $64,9[52,1-75,9]$ & $35,1[24,1-47,8]$ & $48,8[42,7-54,8]$ & $51,2[45,1-57,3]$ \\
\hline $2^{\circ}$ terço $(>1,2 \leq 1,3)$ & $44,5[36,7-52,6]$ & $55,5[47,4-63,3]$ & $25,2[19,3-32,2]$ & $74,8[67,8-80,7]$ \\
\hline $3^{\circ}$ terço $(>1,3)$ & $30,9[24,9-37,7]$ & $69,1[62,3-75,1]$ & $25,1[18,4-33,4]$ & $74,8[66,6-81,6]$ \\
\hline \multicolumn{5}{|l|}{ Proteína $\mathrm{C}$ reativa $(\mathrm{mg} / \mathrm{dL})^{\dagger}$} \\
\hline Normal $(\leq 0,5)$ & $42,6[37,4-48,0]$ & $57,4[52,0-62,6]$ & $37,2[33,0-41,6]$ & $62,8[58,4-67,0]$ \\
\hline Alterada $(>0,5)$ & $18,9[8,2-38,0]$ & $81,1[62,0-91,8]$ & $29,8[20,6-41,0]$ & $70,2[59,0-79,4]$ \\
\hline \multicolumn{5}{|l|}{ Acesso a serviços de saúde (ult. 6 meses) } \\
\hline Não & $44,6[37,4-52,0]$ & $55,4[48,0-62,5]$ & $38,0[28,8-48,3]$ & $61,9[51,7-71,2]$ \\
\hline $\operatorname{Sim}$ & $36,7[29,1-45,0]$ & $63,3[55,0-70,9]$ & $34,2[29,3-39,5]$ & $65,8[60,5-70,6]$ \\
\hline \multicolumn{5}{|l|}{ Diabetes ${ }^{\ddagger}$} \\
\hline Não & $43,1[36,9-49,4]$ & $56,9[50,5-63,0]$ & $40,0[34,9-45,4]$ & $60,0[54,6-65,1]$ \\
\hline $\operatorname{Sim}$ & $35,2[24,0-48,3]$ & $64,8[51,7-76,0]$ & $27,4[20,1-36,3]$ & $72,5[63,7-79,9]$ \\
\hline
\end{tabular}

* Pontos de corte: sexo masculino $\geq 94 \mathrm{~cm}$ e sexo feminino $\geq 80 \mathrm{~cm}$.

${ }^{\dagger}$ valor $p<0,05$ para a estatística $\mathrm{F}$ - sexo masculino; ${ }^{\ddagger}$ valor $p<0,05$ para a estatística $\mathrm{F}$ - sexo feminino.

\section{DISCUSSÃO}

Os resultados do estudo revelaram elevada prevalência de dislipidemia no município, em ambos os sexos, principalmente após os 40 anos de idade. Diferentes conjuntos de variáveis permaneceram no modelo final, destacando-se, entre os homens, "faixas etárias", "renda", "consumo de fibras" e "índice de conicidade" e, entre as mulheres, "faixas etárias", "consumo de ácidos graxos saturados", "média diária de tempo sentado" e "razão cintura-quadril".

Estudos conduzidos em diferentes populações $(20,21)$ revelaram alterações nas frações lipídicas, segundo fatores sociodemográficos. Salinas e cols. (20), com dados da coorte "Cameron County Hispanic
Cohort" (2003-2010), observaram que homens mexicanos $>30$ anos apresentaram maior chance de se tornarem dislipidêmicos, em relação àqueles < 30 anos. Koch e cols. (21) em outro estudo de coorte (1997-2006), com chilenos > 20 anos, identificaram maior chance de dislipidemia em indivíduos com baixa escolaridade e baixo nível socioeconômico. É possível supor que a associação entre renda e dislipidemia seja mediada pelo excesso de peso. Moraes e cols. (22) em estudo epidemiológico conduzido em Ribeirão Preto, em 2006, investigando o estágio atual da transição nutricional no município, identificaram efeito direto da renda sobre o excesso de peso, que pode ser considerado um evento intermediário para a dislipidemia. 
Tabela 5. Razões de prevalências (RP) brutas e ajustadas, com respectivos intervalos de confiança $\left(\mathrm{IC}_{95 \%}\right)$, segundo sexo. Modelos finais. Ribeirão Preto, São Paulo, Brasil, 2007. Projeto EPIDCV

\begin{tabular}{|c|c|c|c|c|}
\hline & $\begin{array}{c}\text { RP } \\
\text { brutas }\end{array}$ & IC $_{95 \%}$ & $\begin{array}{c}\mathbf{R P} \\
\text { ajustadas* }\end{array}$ & $\mathbf{I C}_{95 \%}$ \\
\hline \multicolumn{5}{|l|}{ Masculino } \\
\hline \multicolumn{5}{|l|}{ Faixas etárias } \\
\hline 30-39 anos & 1 & & 1 & \\
\hline 40-49 anos & 1,4 & $1,1-1,8$ & 1,4 & $1,1-1,8$ \\
\hline 50-59 anos & 1,6 & $1,2-2,1$ & 1,6 & $1,1-2,1$ \\
\hline 60 anos e mais & 2,0 & $1,6-2,5$ & 1,9 & $1,4-2,5$ \\
\hline \multicolumn{5}{|l|}{ Renda individual (em R\$) } \\
\hline Sem renda & 1 & & 1 & \\
\hline $1^{\circ}$ terço $(\leq 700,00)$ & 0,8 & $0,7-1,1$ & 1,2 & $0,9-1,6$ \\
\hline $2^{\circ}$ terço $(>700,00 \leq 1.400,00)$ & 1,0 & $0,8-1,2$ & 1,5 & $1,2-1,9$ \\
\hline $3^{\circ}$ terço $(>1.400,00)$ & 0,8 & $0,7-1,0$ & 1,3 & $1,1-1,7$ \\
\hline \multicolumn{5}{|l|}{ Consumo de fibras (em g/dia) } \\
\hline $1^{\circ}$ terço $(\leq 24,7)$ & 1 & & 1 & \\
\hline $2^{\circ}$ terço $(>24,7 \leq 42,2)$ & 1,3 & $1,1-1,3$ & 1,3 & $1,1-1,6$ \\
\hline $3^{\circ}$ terço $(>42,2)$ & 1,1 & $0,9-1,4$ & 1,1 & $0,9-1,4$ \\
\hline \multicolumn{5}{|l|}{ Índice de conicidade } \\
\hline $1^{\circ}$ terço $(\leq 1,2)$ & 1 & & 1 & \\
\hline $2^{\circ}$ terço $(>1,2 \leq 1,3)$ & 1,6 & $1,1-2,3$ & 1,5 & $0,9-2,4$ \\
\hline $3^{\circ}$ terço $(>1,3)$ & 2,0 & $1,4-2,8$ & 1,5 & $1,1-2,4$ \\
\hline
\end{tabular}

Faixas etárias

\begin{tabular}{|c|c|c|c|c|}
\hline 30-39 anos & 1 & & 1 & \\
\hline 40-49 anos & 1,7 & $1,3-2,3$ & 1,6 & $1,3-2,2$ \\
\hline $50-59$ anos & 2,4 & $1,9-3,2$ & 2,2 & $1,7-3,0$ \\
\hline 60 anos e mais & 2,4 & $1,8-3,0$ & 1,9 & $1,4-2,6$ \\
\hline \multicolumn{5}{|c|}{ Ácidos graxos saturados (em g/dia) } \\
\hline $1^{\circ}$ terço $(\leq 19,2)$ & 1 & & 1 & \\
\hline $2^{\circ}$ terço $(>19,2 \leq 24,8)$ & 1,0 & $0,8-1,1$ & 0,9 & $0,7-1,0$ \\
\hline $3^{\circ}$ terço $(>24,8)$ & 0,8 & $0,7-0,9$ & 0,8 & $0,7-0,9$ \\
\hline \multicolumn{5}{|l|}{$\begin{array}{l}\text { Média diária de tempo sentado } \\
\text { (em minutos) }\end{array}$} \\
\hline $1^{\circ}$ terço $(\leq 180,0)$ & 1 & & 1 & \\
\hline $2^{\circ}$ terço $(>180,0 \leq 308,6)$ & 0,9 & $0,8-1,0$ & 0,9 & $0,7-1,0$ \\
\hline $3^{\circ}$ terço $(>308,6)$ & 1,2 & $1,1-1,3$ & 1,2 & $1,1-1,4$ \\
\hline \multicolumn{5}{|l|}{ Razão cintura-quadril } \\
\hline $1^{\circ}$ terço $(\leq 0,8)$ & 1 & & 1 & \\
\hline $2^{\circ}$ terço $(>0,8 \leq 0,9)$ & 1,5 & $1,3-1,8$ & 1,4 & $1,1-1,7$ \\
\hline $3^{\circ}$ terço $(>0,9)$ & 1,6 & $1,4-2,0$ & 1,2 & $1,0-1,6$ \\
\hline
\end{tabular}

* Foram retidas no modelo final as variáveis: acesso a serviços de saúde e diabetes melito.

No Brasil, grande parte dos estudos epidemiológicos relacionados às doenças crônicas não tem sido conduzida em base populacional. Em diferentes estudos
$(23,24)$ com participantes $>18$ anos, os autores detectaram, respectivamente, prevalências de dislipidemias (40\% e 15,9\%) inferiores, em magnitude, às do presente estudo. A falta de representatividade de algumas dessas amostras ou os métodos utilizados para a mensuração da dislipidemia (24) podem ter comprometido a validade interna desses estudos e/ou subestimado a prevalência do desfecho em relação aos achados de Ribeirão Preto.

Em estudo epidemiológico de base populacional, conduzido recentemente nesse município, os autores identificaram elevada prevalência de diabetes melito (25). $\mathrm{Se}$, por um lado, o diabetes pode contribuir para a dislipidemia, em contrapartida, no presente estudo, os pontos de corte de cada uma das frações lipídicas CT, HDL-c e LDL-c, correspondentes aos percentis 25,50 e 75 , foram apenas discretamente superiores nos diabéticos, em relação aos não diabéticos. Parece plausível supor, portanto, que a elevada prevalência do desfecho ultrapassou a capacidade discriminatória de exposições relevantes como o diabetes na "predição" da dislipidemia.

Em relação às variáveis comportamentais, resultados controversos têm sido descritos sobre a associação entre "tempo sentado" e dislipidemia. No modelo final do presente estudo, as participantes do sexo feminino, classificadas no último terço da média diária de tempo sentado (> 308,60 minutos/dia), apresentaram prevalência de dislipidemia $20 \%$ maior do que aquelas classificadas no primeiro terço. Thorp e cols. (26), avaliando dados da coorte "The Australian Diabetes, Obesity and Lifestyle (AusDiab)" (1999-2005), em amostra de 4.684 adultos $>25$ anos, observaram, em ambos os sexos, aumento médio significativo de $0,01 \mathrm{mmol} / \mathrm{L}$ nos níveis de triglicérides para cada hora/dia de aumento do tempo sentado, mesmo após ajustamento para circunferência da cintura e atividade física no lazer. Em contrapartida, Heer e cols. (27), em estudo transversal aninhado em coorte (2004-2010) com 11.268 hispânicos > 18 anos, não observaram associação entre “tempo sentado" e "colesterol elevado". É possível que essas diferenças se devam, em parte, aos métodos empregados para as dosagens de lipídios e/ou ao tipo de instrumento utilizado para mensurar o "tempo sentado". No estudo de Heer e cols. (27), por exemplo, a hipercolesterolemia foi aferida por meio de entrevistas, técnica que apresenta baixa sensibilidade em relação às dosagens bioquímicas.

Em relação ao padrão de atividade física, Lee e cols. (28), em estudo de coorte entre 1979-2006 que arrolou 
participantes $>18$ anos, observaram que os indivíduos que mantiveram ou melhoraram o padrão de atividade física apresentaram menor risco de desenvolver hipercolesterolemia quando comparados àqueles que diminuíram o padrão de atividade física. Monda e cols. (29), na primeira visita de seguimento da coorte do "Atherosclerosis Risk in Communities (ARIC) Study" (1987-1989), com o objetivo de avaliar os efeitos da atividade física sobre o perfil lipídico, em adultos americanos entre 45 e 64 anos, observaram que um aumento de 180 METs$\mathrm{min} / \mathrm{semana}$ promoveu melhora no padrão lipídico para CT, LDL-c, HDL-c e TG, em homens e mulheres.

Entre as limitações do presente estudo, destaca-se o delineamento transversal, no qual aferições simultâneas de exposições e desfecho podem comprometer a direcionalidade das associações, conduzindo ao viés de causalidade reversa. Sob tais circunstâncias, os achados do estudo revelaram, em ambos os sexos, prevalências de dislipidemias de menor magnitude entre os que consumiram maior quantidade de gordura saturada e que apresentaram maior consumo energético (em kcal/ dia), contrapondo-se àqueles que consumiram maior quantidade de fibras e praticaram atividade física (sexo masculino) nos quais a dislipidemia apresentou maior prevalência. Parece plausível supor, por conseguinte, que pessoas com alterações lipídicas devem estar sendo mais estimuladas a praticar atividade física e a adotar dietas saudáveis do que as demais. Outra limitação importante é o vício de recordação e a baixa sensibilidade dos inquéritos nutricionais de frequência para a aferição de hábitos alimentares, embora tenham sido utilizadas, no estudo, as equações propostas por Willett (14) para o ajustamento do consumo alimentar, segundo o total calórico da dieta.

Grande parte dos estudos relacionados ao padrão dietético versus dislipidemias tem sido conduzida a partir de ensaios clínicos randomizados (30). Entretanto, mesmo se considerando as vantagens de tais estudos sobre os observacionais, no que diz respeito ao fato de que a randomização permite o ajustamento simultâneo para fatores de confusão, conhecidos ou não, o reduzido tamanho de amostras nesses ensaios limita inferências de seus resultados para a população geral.

Ainda em relação aos fatores comportamentais, os resultados do presente estudo evidenciaram, na fase descritiva, relação direta entre a duração do hábito de fumar e a dislipidemia e associação estatisticamente significante, no sexo feminino, embora esta variável não tenha permanecido no modelo final.
Em relação aos indicadores antropométricos, os resultados do estudo evidenciaram associações independentes com a dislipidemia do índice de conicidade, entre os homens, e da razão cintura/quadril, entre as mulheres, destacando-se a relevância da obesidade central, em relação ao desfecho, em ambos os sexos.

A literatura tem evidenciado associação entre distribuição de gordura corporal e dislipidemias $(31,32)$. Contudo, existem divergências sobre quais indicadores antropométricos para aferição da obesidade global ou central seriam os melhores preditores daquele desfecho. Wietlisbach e cols. (31), ao avaliarem dados de uma coorte (1990-1997) com indivíduos entre 35-64 anos de 31 regiões que incluíram América do Norte, Europa, Ásia e Oceania, observaram que o IMC, entre os homens, e a CC, entre as mulheres, estiveram associados à dislipidemia, mesmo após ajustamento para idade, região e tabagismo. Knowles e cols. (32), em estudo transversal (2009), com adultos peruanos > 18 anos, observaram que indivíduos com valores elevados de IMC e CC apresentaram maior chance de níveis altos de triglicerídeos.

No que pesem algumas diferenças, em relação à literatura, quanto ao delineamento do estudo, aos métodos de aferição de exposições e desfecho, à representatividade das amostras, aos diferentes conjuntos de variáveis de confusão consideradas, bem como às técnicas aplicadas para análise dos dados, a persistência da associação entre os indicadores de obesidade central e a dislipidemia reforça a importância dessas exposições sobre o desfecho aqui considerado.

Na última década, têm sido cada vez mais frequentes os estudos epidemiológicos que investigam a associação entre inflamação e doenças crônicas ou seus fatores de risco, sendo reconhecida como determinante para o desenvolvimento e a progressão da aterosclerose $(33,34)$. García-Lorda e cols. (33) em 2001, utilizando dados da Encuesta de Salud de Catalunya-ESCA, que envolveu espanhóis > 18 anos, identificaram que o IMC e os níveis de triglicerídeos estiveram associados aos níveis de PCR-U, no sexo feminino e, no masculino, permaneceram associados a CC, níveis de triglicerídeos e fração HDL-c. À PCR-U tem sido atribuído o importante papel de modulador do risco de eventos coronarianos agudos (infarto incidente e morte súbita), ao se considerar os escores de risco de Framinghan como variáveis preditoras em modelos multivariados (34). No presente estudo e, em ambos os sexos, embora a prevalência de dislipidemia tenha sido bem mais elevada entre os parti- 
cipantes com PCR-U alterada (fase descritiva), essa variável não permaneceu nos modelos finais, após o ajustamento simultâneo para os demais fatores de confusão.

Torna-se importante destacar que, em Ribeirão Preto, optou-se por considerar o número de componentes alterados para a definição de dislipidemia, em vez da prevalência de cada um deles, isoladamente, tendo em vista que $42,4 \%$ da amostra apresentaram alterações em duas ou mais frações lipídicas. Sob tais condições, a rarefação de participantes com alteração de apenas uma das frações, aliada ao imperativo de estratificação por sexo, limitaria o poder estatístico do estudo.

Considera-se oportuno enfatizar que, por outro lado, na ausência de informações disponíveis sobre o uso de medicamentos para o controle da dislipidemia, optou-se por incluir, nos modelos finais, o ajustamento para "acesso a serviços de saúde", que, embora indiretamente, pode ter contribuído para ajustar tais modelos para o uso de medicamentos.

Em suma, no que pesem as limitações aqui mencionadas, o rigor metodológico com que foram conduzidas as diferentes etapas do estudo bem como a elevada taxa de resposta $(>80 \%)$, ao lado do acordo obtido para os indicadores de reprodutibilidade das informações (estatística Kappa > 0,80), entre outros aspectos, reforçam a validade interna do estudo e indicam que os achados não foram decorrentes de vícios sistemáticos de seleção ou confusão.

As doenças cardiovasculares têm sido consideradas a principal causa de mortalidade em Ribeirão Preto e a dislipidemia, fator independentemente associado a esse desfecho (35). Os resultados do presente estudo indicam que a elevada prevalência de dislipidemia no município de Ribeirão Preto pode ser considerada um problema de Saúde Pública e que fatores sociodemográficos, sedentarismo, fatores dietéticos e obesidade central constituem-se em fatores independentemente associados ao desfecho.

Conclui-se que políticas públicas de intervenção direcionadas à adoção de hábitos saudáveis de consumo alimentar, bem como ao controle da obesidade central e do sedentarismo, poderão contribuir para o declínio da prevalência de dislipidemias, esperando-se que tais medidas possam reduzir os indicadores de morbimortalidade por doenças cardiovasculares no município, tendo em vista o envelhecimento populacional progressivo de sua população.

Contribuição dos autores: Suzana Alves de Moraes - Elaborou a proposta do estudo, colaborou no gerenciamento e análise dos dados, interpretação e discussão dos resultados, elaboração e revisão de todas as versões do manuscrito. Michele Vantini Checchio - Participou da elaboração da proposta do estudo, colaborou no levantamento bibliográfico, na elaboração das tabelas de resultados, na discussão dos resultados e na revisão da versão final do manuscrito. Isabel Cristina Martins de Freitas - Participou da elaboração da proposta do estudo, colaborou no gerenciamento e na análise dos dados, discussão dos resultados e revisão de todas as versões do manuscrito.

Agradecimentos: projeto EPIDCV: Financiado pela Fundação de Amparo à Pesquisa do Estado de São Paulo (Fapesp) (Processo n ${ }^{\circ}$ 2006/50495-2); Michele Vantini Checchio - Bolsa de Pós-Graduação - Demanda Social-Capes; Isabel Cristina Martins de Freitas - Bolsa de Pós-Doutorado Fapesp (Processo $n^{\circ}$ 2012/51141-0). Os autores agradecem às Agências Financiadoras descritas.

Declaração: os autores declaram não haver conflitos de interesse científico neste estudo.

\section{REFERÊNCIAS}

1. Popkin BM. Introduction. In: Caballero B, Popkin BM (ed.). The nutrition transition: diet and disease in the developing world. London: Academic Press; 2002. p. 16-21.

2. Noncommunicable Diseases and Mental Health, World Health Organization. Integrated management of cardiovascular risk: report of a WHO meeting, Geneva 9-12, July 2002.

3. Moraes SA, Suzuki CS, Freitas ICM, Costa ML Jr. Mortalidade por doenças do aparelho circulatório no município de Ribeirão Preto, SP, de 1980 a 2004. Arq Bras Cardiol. 2009;93(6):637-44.

4. Diaz MN, Frei B, Vita JA, Keaney JF Jr. Antioxidants and atherosclerotic heart disease. N Engl J Med. 1997;37(6):408-16.

5. Wang S, Xu L, Jonas JB, You QS, Wang YX, Yang H. Prevalence and associated factors of dyslipidemia in the adult Chinese population. PloS ONE. 2011;6(3):e17326.

6. Rajaram S, Haddad EH, Mejia A, Sabaté J. Walnuts and fatty fish influence different serum lipid fractions in normal to mildly hyperlipidemic individuals: a randomized controlled study. Am J Clin Nutr. 2009;89:(Suppl):1657S-63S.

7. Cetin I, Yildirim B, Sahin S, Sahin I, Etikan I. Serum lipid and lipoprotein levels, dyslipidemia prevalence, and the factors that influence these parameters in a Turkish population living in the province of Tokat. Turk J Med Sci. 2010;40(5):771-82.

8. KaoT-W, Lu I-S, Liao K-C, Lai H-Y, Loh C-H, Kuo H-K. Associations between body mass index and serum levels of $\mathrm{C}$-reactive protein. S Afr Med J. 2009;99:326-30.

9. Silva NN. Amostragem probabilística: um curso introdutório. 2.ed. São Paulo: EDUSP; 2001.

10. Instituto Brasileiro de Geografia e Estatística (IBGE). Censo Demográfico 2000. Rio de Janeiro, 2000.

11. Grundy SM, Cleeman JI, Merz CN, Brewer HB Jr, Clark LT, Hunninghake DB, et al. Implications of recent clinical trials for the National Cholesterol Education Program Adult Treatment Panel III guidelines. Circulation. 2004;110:227-39.

12. Craig $C$, Marshall $A$, Sjostrom $M$, Bauman AE, Booth $M L$, Pratt $M$, et al. International physical questionnaire: 12-country reliability and validity. Med Sci Sports Exerc. 2003;35:1381-95.

13. Fornés NS, Martins IS, Velásquez-Meléndez G, Latorre MRDO. Escores de consumo alimentar e níveis lipêmicos em população de São Paulo, Brasil. Rev Saúde Pública. 2002:36:12-8. 
14. WillettWC, Howe GR, Kushi LH. Adjustment for total energy intake in epidemiologic studies. Am J Clin Nutr. 1997;65(4 suppl):1220S8S; discussion 1229S-31S.

15. World Health Organization. Obesity: preventing and managing the global epidemic: report of a WHO Consultation. Geneva Switzerland: World Health Organization; 2000. (Who technical report series 894).

16. Alberti G, Zimmet P, Shaw J, Grundy SM. The IDF consensus worldwide definition of the metabolic syndrome. Brussels: International Diabetes Federation; 2006.

17. Valdez R, Seidell JC, Ahn Yl, Weiss KM. A new index of abdominal adiposity as an indicator of risk for cardiovascular disease. A cross-population study. Int J Obes. 1992;16:77-82.

18. World Health Organization. Report of aWHO Consultation. Definition, diagnosis and classification of diabetes mellitus and its complications. Part I: diagnosis and classification of diabetes mellitus. Geneva: World Health Organization: 1999. (WHO/NCD/NCS/99.2).

19. Barros AJD, Hirakata VN. Alternatives for logistic regression in cross-sectional studies: an empirical comparison of models that directly estimate the prevalence ratio. BMC Med Res Methodol. 2003;3:21.

20. Salinas J, McCormick JB, Rentfro A, Hanis C, Hossain MM, Rahbar $\mathrm{MH}$, et al. The missing men: high risk and low use of health care in men of Mexican origin. Am J Mens Health. 2011;5(4):332-40.

21. Koch $E$, Romero T, Manríquez $L$, Paredes $M$, Ortúzard $E$, Taylor $A$, et al. Desigualdad educacional y socioeconómica como determinante de mortalidad em Chile: análisis de sobrevida em la cohorte del proyecto San Francisco. Rev Méd Chile. 2007;135:1370-9.

22. Moraes SA, Humberto JSM, Freitas ICM. Estado nutricional e fatores sociodemográficos em adultos residentes em Ribeirão Preto, SP, 2006. Projeto OBEDIARP. Rev Bras Epidemiol. 2011;14(4):662-76.

23. Martinez TLR, Santos RD, Armaganijan D, Torres KP, Loures-Vale $A$, Magalhães ME, et al. National alert campaign about increased cholesterol. Determination of cholesterol levels in 81,262 Brazilians. Arq Bras Cardiol. 2003;80(6):635-8.

24. Malta DC, Moura EC, Moraes Neto OL. Gender and schooling inequalities in risk and protective factors for chronic diseases among Brazilian adults, through telephone survey. Rev Bras Epidemiol. 2011;14(1 Supl.):125-35.

25. Moraes SA, Freitas ICM, Gimeno SGA, Mondini L. Prevalência de diabetes mellitus e identificação de fatores associados em adultos residentes em área urbana de Ribeirão Preto, São Paulo, Brasil, 2006: Projeto OBEDIARP. Cad Saúde Pública. 2010;26:929-41.

26. Thorp AA, Healy GN, Owen N, Salmon J, Ball K, Shaw JE, et al. Deleterious associations of sitting time and television viewing time with cardiometabolic risk biomarkers: Australian Diabetes, Obesity and Lifestyle (AusDiab) study 2004-2005. Diabetes Care. 2010;33(2):327-34.

27. Heer HD, Wilkinson AV, Strong LL, Bondy ML, Koehly LM. Sitting time and health outcomes among Mexican origin adults: obesity as a mediator. BMC Public Health. 2012;12:896.

28. Lee D, Sui X, Church TS, Lavie CJ, Jackson AS, Blair SN. Changes in fitness and fatness on the development of cardiovascular disease risk factors: hypertension, metabolic syndrome and hypercolesterolemia. J Am Coll Cardiol. 2012;59(7):665-72.

29. Monda KL, Ballantyne CM, North KE. Longitudinal impact of physical activity on lipid profiles in middle-age adults: The Atherosclerosis Risk in Communities Study. J Lipid Res. 2009;50:1685-91.

30. Koh KK, Quon MJ, Shin KC, Lim S, LeeY, Sakuma I, et al. Significant differential effects of omega- 3 fatty acids and fenofibrate in patients with hypertriglyceridemia. Atherosclerosis. 2012;537-44.

31. Wietlisbach V, Marques-Vidal $P$, Kuulasmaa $K$, Karvanen J, Paccaud F; WHO MONICA Project. The relation of body mass index and abdominal adiposity with dyslipidemia in 27 general populations of the WHO MONICA Project. Nutr Metab Cardiovasc Dis. 2013;23(5):432-42. doi: 10.1016/j.numecd.2011.09.002. Epub 2011 Dec 30.

32. Knowles KM, Paiva LL, Sanchez SE, Revilla L, Lopez T, Yasuda $M B$, et al. Waist circumference, body mass index, and other measures of adiposity in predicting cardiovascular risk factors among Peruvian adults. Int J Hypertens. 2011;2011:931402. doi:10.4061/2011/931402.

33. García-Lorda P, Bulló M, Balanza R, Salas-Salvadó. C-reactive protein, adiposity and cardiovascular risk factors in a Mediterranean population. Int J Obesity. 2006;30:468-74.

34. Koenig W, Löwel H, Baumert J, Meisinger C. C-reactive protein modulates risk prediction based on the Framinghan Score: implications for future risk assessment: results from a large cohort study in southern Germany. Circulation. 2004;109:1349-53.

35. Moraes SA, Freitas ICM. Doença isquêmica do coração e fatores associados em adultos de Ribeirão Preto, SP. Rev Saúde Pública. 2012;46(4):591-601. 